\title{
Impact of processing load on analogical mapping with visual sequences in children with developmental language disorders (DLD)
}

\author{
Krzemien Magali $^{\mathrm{a}}$, Maillart Christelle ${ }^{\mathrm{a}}$, Parisse Christophe, and Leroy Sandrine ${ }^{\mathrm{a}}$ \\ ${ }^{a}$ Department of Speech and Language Therapy, Research Unit on Childhood, University of \\ Liège, B38, 30 rue de l'Aunaie, 4000 Liège, Belgium \\ ${ }^{b}$ Modyco-Inserm, University of Paris Ouest Nanterre la Défense, Nanterre, France
}

Correspondence: Sandrine Leroy, Sandrine.Leroy@uliege.be

\begin{abstract}
Background: Analogical mapping is a domain-general cognitive process which is notably used in language development, and particularly in the abstraction of construction schemas. Children with developmental language disorders (DLD) display an impairment in linguistic productivity and creativity, which can be linked to a lack of generalization of construction schemas.
\end{abstract}

Aims: The current study aimed at investigating analogical mapping in children with DLD, and especially the influence of processing load, as it could explain the lack of creativity observed in children with DLD. We hypothesized that analogical mapping is altered in children with DLD and that greater cognitive load (sequential presentation and no perceptual support) would be linked to poorer performance in these children.

Methods and procedures: Fifteen children with DLD and their age-matched peers were administrated a visual analogical reasoning task where children have to complete a sequence sharing the same relational structure as previously presented sequences. Two factors influencing processing load were studied: the modality of presentation (sequential vs. simultaneous) and the perceptual support (with vs. without).

Outcomes and results: Results showed an expected group effect with poorer performance in children with DLD compared to children with TLD. Results corroborated hypotheses according to which children with DLD have difficulties with analogical mapping, which could hinder their abstraction of construction schemas. Results about the influence of processing load were mixed. While the difference between the two groups was more marked for the items without perceptual support than for the items with perceptual support, children with DLD were not more affected by the sequential presentation than children with TLD.

Conclusions and implications: Children with DLD have impaired analogical mapping competences, especially when the relational similarities are not supported by perceptual cues. This impairment may be the cause of their difficulties in abstracting construction schemas, thus 
provoking their poor linguistic productivity and creativity. However, more studies are needed to confirm this hypothesis, as the influence of analogical reasoning on language development could also be reversed or could be linked to another external factor.

\section{What this paper adds}

1. Analogical reasoning is involved in language development, notably in the abstraction of construction schemas (Bybee 2010, Gentner and Namy 2006). Analogical reasoning is impaired in children with developmental language disorders (DLD) in linguistic or non-linguistic tasks (Krzemien et al. 2017, Leroy et al. 2014). We make the assumption that the analogical reasoning impairment observed in children with DLD is linked to a difficulty in processing analogical tasks with a high cognitive load, which could cause their language disorders.

2. Children with DLD have worse performance than their age-matched peers without language disorders in a pattern-based abstraction task, involving analogical mapping. High cognitive load has a greater impact on their performance, but only with one of the two variables manipulated: children with DLD are not more impaired by sequential presentation of items than their peers. In contrast, they have difficulty in solving analogies when no perceptual cues support the relational similarities, while control children are not influenced by this variable.

3. It is possible that a deficit in pattern-based abstraction, which is fundamental to language productivity (Gómez and Gerken 2000), causes the languages disorders observed in DLD. Moreover, it seems that this deficit is linked to a difficulty in processing the items with high processing load when it is due to the absence of perceptual cues. However, more studies are needed to confirm this link, as the influence between analogical reasoning and language is complex and could be mutual or imply other factors. This study therefore brings data about a deficit in children with DLD in a domain-general function linked to language development, which could lead to better comprehension and intervention regarding DLD.

\section{Introduction}

\section{Analogical reasoning in cognitive and language development}

Analogical reasoning is a domain-general cognitive process which plays a central role in human cognition and is tightly related to general fluid intelligence (Chuderski 2015). It is omnipresent in the daily life and it is used in any cognitive process, as soon as it requires the abstraction of a schema (Gentner and Smith 2013). For instance, analogies help solve current problems based on problems solved in the past (Chen 1996). They are also useful for understanding metaphors, and especially metaphors based on relational similarities (such as in "a window is like an eye", Gentner 1988). Besides that, analogies are used in education (for 
example when a professor draws an analogy between two historical events to improve their conceptualization, Richland and Simms 2015), in argumentation (such as in advertising, Roehm and Sternthal 2001) and in social judgment (for example, when someone thinks about a person while comparing him/her to someone else or to him/herself, Mussweiler and Epstude 2009). Although analogies vary widely in their appearance, content and usage, the core process required for reasoning by analogy, which is called mapping (Gentner and Markman 1997), is common to analogical reasoning of all types, whatever the nature of the task. Gentner (1983) describes this process in her structure-mapping theory. Analogical mapping involves two steps. During the first step, analogical mapping involves a structural alignment between two situations, based on a common relational structure (Gentner and Markman 1997). An overlap between the relational structures of the two situations being analogized is essential, whereas concrete property matches between them are not necessary. Moreover, according to the systematicity principle, large connected systems of higher-order relations will be preferred to lower order matches (Gentner and Smith 2013). The second step of analogical mapping is projecting inferences while extending the relational structure of a previous situation onto the new situation, which yields to a better knowledge of this new situation. The inferences are candidate inferences which will require evaluation to ensure their veracity (Gentner and Smith 2013). For example, we can draw an analogy between the metabolism of a cell and the fire, aligning elements such as the fact that they both produce energy with oxygen. Knowing that fire releases water and carbon dioxide, we can infer that cells do too (Gentner and Colhoun 2010).

Besides its role in general cognitive development, analogical mapping, and especially the process of structural alignment, is required in prior and in later language development (for a review Gentner and Namy 2006). First, comparison, which involves structural alignment, is central to categorize lexical items, especially when categories are defined by relational properties, such as relational nouns or verbs (Childers 2011, Gentner et al. 2011): comparing several exemplars of a new word allows children to identify relational similarities between those exemplars and to extend this new word appropriately, without focusing on misleading perceptual information. Moreover, in morphosyntactic development, analogical mapping is considered to be a key component in the abstraction of construction schemas (e.g. Bybee 2010). Constructions are defined by Goldberg (2003, p. 219) as "stored pairings of form and function", whose "form or function is not strictly predictable from its component parts or from other constructions recognized to exist". Constructions vary in complexity and in abstractness: while idioms are concrete constructions, the plural or passive in English are considered as abstract ones (Tomasello 2009). Analogical mapping is used to align different forms associated to their function, and to abstract the common underlying construction. It also allows the use of a new item in a specific construction. For example, children can abstract the general past tense construction of English and apply it to a new verb thanks to this mapping (Bybee 2010). Analogical mapping, and structural alignment, therefore allow children to abstract constructions from concrete forms and to produce novel sentences, applying novel lexical items to those abstracted constructions (Bybee 2010, Tomasello 2009). Thus, it allows for productivity and creativity in language, the aspects of language development that are at risk in children with developmental language disorders (DLD). 


\section{Analogical reasoning and developmental language disorders}

The expression developmental language disorders refers to a developmental linguistic pathology in which children present a slow development of spoken language in spite of normal hearing, normal motor development, and the absence of neurodevelopmental disorders (such as autism) or intellectual and emotional impairments (Schwartz 2009). Children with DLD obtain age-appropriate scores on non-verbal tests of intelligence (Schwartz 2009), but their performance can be lower than the one of their peers (Earle et al. 2017, Vugs et al. 2013). The morphosyntactic domain is particularly impaired in children with DLD, as they have difficulty in generalizing linguistic knowledge, which impedes the productive use of morphemes and syntactic structures. They encounter important difficulty in verbal morphology, and especially in the past tense use (Christensen and Hansson 2012, Tomas et al. 2017). They also seem to have difficulty using nominal morphology (Conti-Ramsden and Windfuhr 2002) and syntax (Skipp et al. 2002) productively: they are less able than their peers to apply a morpheme or a syntactic structure to novel words. Children with DLD have a lack of syntactic creativity and are more input dependent than children with typical language development (TLD) (for a review Riches et al. 2006). They tend to use forms that they have already heard (Conti-Ramsden and Windfuhr 2002, Hsu and Bishop 2010). These observations are compatible with the hypothesis of a lack of generalization of construction schemas in children with DLD. Given the role of analogical mapping in the abstraction of construction schemas and the generalization disorders of children with DLD, we decided to investigate the integrity of analogical mapping in children with DLD. An analogical mapping impairment could indeed be responsible for their language disorders and especially for their difficulty in generalization and in linguistic productivity.

Several studies have explored the impact of language disorders on analogical reasoning (for a review Leroy et al. 2012), suggesting that children with DLD perform less well than children with TLD because of their language impairment. Masterson et al. (1993) found that children with DLD perform less well than age-matched children but as well as languagematched children in verbal analogy tasks. The authors conclude that linguistic abilities are a better predictor of analogical performance than cognitive ones. Until recently, the reverse influence of analogical mapping on language disorders has not been envisaged. However, given the role of analogies on language development (Bybee 2010), a potential analogical reasoning impairment causing language disorders in DLD should be investigated. Krzemien et al. (2017) used a scene analogy task (Richland et al. 2006) where children have to associate two characters sharing the same relational role in two different situations presented visually and simultaneously. Children with DLD have a lower performance than age-matched children. They also have the same global performance as language-matched children. Leroy et al. (2012) studied the integrity of analogical mapping in children with DLD, namely their abilities to infer a relational structure and generalize this structure to new items. They used a pattern-based abstraction task. Pattern-based abstraction, which can be described in terms of "relational operations over physical stimuli in sequence" (Gómez and Gerken 2000), is used by children for acquiring structured sequence regularities, including word order regularities in addition to 
words. Children use these regularities for developing their language. In their task, children had to compare two sequences composed of geometric shapes and had to complete a third sequence by choosing missing geometric shapes. Results showed that the performance of children with DLD was poorer than that of age-matched children with TLD. The authors used a visual task presenting cognitive constraints that were as close as possible to the constraints found in language processing. Consequently, geometric shapes were presented successively. Leroy et al. (2014a) reproduced this protocol comparing linguistic and non-linguistic items. In the linguistic task, children were asked to complete a sequence of non-sense syllables using two sequences previously heard. In the non-linguistic task, they had to do the same with visual forms without semantic content. Here again, children with DLD perform less well than age-matched peers in the two tasks. In this experiment too, items were presented sequentially in order to be as close as possible to language processing. Interestingly, the fact that the group difference was not limited to the linguistic modality suggested that linguistic abilities are not sufficient to predict analogical performance.

To conclude, analogical mapping is a crucial cognitive function which is used to develop a productive and creative language. In lexicon, several exemplars of the same category are mapped in order to abstract this category and to apply it to novel instances (Gentner et al. 2011, Gentner and Namy 1999). In morphosyntax, analogical mapping allows for the generalization of construction schemas: children abstract linguistic structures from the concrete forms that they hear and they can apply these structures to other words in order to produce novel forms (Bybee 2010, Tomasello 2009). Analogical mapping and language are therefore tightly related, the former allowing for the development of the latter. Yet, analogical mapping is impaired in children with DLD (Krzemien et al. 2017, Leroy et al. 2012, Leroy et al. 2014a). Moreover, these children display generalization difficulties, especially in morphosyntax: they struggle to abstract linguistic structures from the input and to produce novel forms (Hsu and Bishop 2010). It is therefore possible that their analogical mapping impairment impedes them to develop a productive language, which would lead to their generalization difficulties and so to their language disorders. In this study, we want to investigate this hypothesis and to try to explain the analogical mapping impairment by manipulating a factor which could be responsible for the difficulties of children with DLD, i. e., processing load.

\section{Analogical reasoning and processing load}

Sequential presentation requires an extra processing load compared to simultaneous presentation. Children have to retain in memory the different sequences and have to process them in order to infer another sequence which shares the same relational structure. Yet, the detection of relational similarity is negatively correlated with the processing load of a task (Gentner and Smith 2012). Richland et al. (2006) found that complicating the relations to be processed (i.e. increasing relational complexity) causes a decrease in participants' performance: children from age 3 to 14 perform better for two-element relations (the cat chases the mouse) than for three-element relations (the dog chases the cat chasing the mouse). Adding perceptual distracting information also leads to a decrease in performance (Richland et al. 2006, Thibaut 
et al. 2010). Thibaut et al. (2010) used a task in which perceptual noise was present or not. Perceptual noise is represented as textures added to the shapes presented but which should not be processed to solve the analogies. Items containing perceptual noise were less accurately identified than other items. They were also processed slower, especially for 8-year-old children.

Children with DLD have processing limitations (Im-Bolter et al. 2006), notably characterized by a working memory deficit (Marton and Schwartz 2003). They experience considerable difficulty when a task is overloaded. They are known to have reduced cognitive resources to allocate to ongoing processing, which may affect their performance, all the more when the cognitive demand of the task is greater than the resources available. This also seems to be true in analogical reasoning tasks. Krzemien et al. (2017) found that the performance of DLD children were more diminished by the increase of relational complexity than languagematched control children. Consequently, the sequential presentation used in Leroy et al. (2012, 2014a) could have overloaded the cognitive resources of children with DLD, thus leading to poorer performance compared to their age-matched peers.

Although object matches are not necessary for carrying out analogical mapping between two situations, the presence of perceptual commonalities between the two situations being analogized can help discover relational similarity and reduce memory load. Gentner and Markman (1997) use the term transparency to refer to the degree of similarity between corresponding objects. In a high-transparency analogy, objects that play the same roles in the common relational structure are highly similar. The authors consider that it is more reliably retrieved from memory and is processed faster than a low-transparency analogy (Waltz et al. 2000). This is particularly true for children with DLD. Leroy et al. (2012) found that all children had better performance when perceptual similarities supported relational ones. However, it is especially the case for children with DLD whose performance dropped dramatically when perceptual similarities between the elements of the sequence decreased. In another experiment, the authors (Leroy et al. 2014a) showed that, while age-matched control children are not influenced by the presence or absence of perceptual similarities, DLD children perform less well when there is no perceptual similarity supporting relational one in a linguistic analogical task. Thus, the absence of perceptual support in analogies seems to overload the cognitive resources of children with DLD, making their performance poorer.

\section{Aim of the study}

The present study aimed to investigate analogical mapping in children with DLD, and more particularly the influence of processing load on their performance. Overall, as shown by Leroy et al. (2012), we predicted that children with DLD would have more difficulty than agematched children with TLD with analogical mapping. We further predicted that even if the performance of all children was affected by an increase in the cognitive load of the task, the difference between children with DLD and children with TLD would be more marked with a greater loaded task involving sequential presentation (vs. simultaneous presentation) and no perceptual similarity (vs. presence of perceptual similarity). 


\section{Methods}

\section{Participants}

Twenty French-speaking children with DLD (2 girls and 18 boys; aged 7;01 to $13 ; 01)$ participated in the study. They were recruited in a "language class" attached to a primary school for children with special needs, in the French-speaking part of Belgium - language class is defined as a class adapted to children with DLD where the educational focus is on the development of language skills. Prior to the study, children with DLD were diagnosed as presenting DLD by speech-language pathologists and child neurologists. All children with DLD had a non-verbal intellectual quotient of 82 or more (non-verbal IQ was measured by using the Performance Intelligence Quotient (PIQ) of the Wechsler Nonverbal Scale of Ability (WNV), Wechsler and Naglieri 2006). Moreover, they had normal hearing, vision, oral and speech motor abilities. Finally, children with DLD scored less than -1.25 SD below the expected normative performance in at least 2 language components (according to the DLD criteria adopted by Leonard et al. 2007), most notably in the productive grammatical abilities. The children's language abilities were assessed by five subtests of the Evaluation du Langage Oral, a test frequently used by French speech-language therapists (ELO: French Language Evaluation Khomsi 2001): lexical production (picture naming); lexical reception (picture designation); phonological production (word repetition); morphosyntactic production (utterance production); and morphosyntactic reception (picture designation).

Twenty children with TLD (11 girls and 9 boys) aged from 7;05 to 12;09 were recruited from schools in the French-speaking part of Belgium. The same medical history questionnaire was completed by the parents. Their linguistic performances were controlled with the same subtests as the children with DLD and all of them scored average or above average for their age (performance higher than -1 SD). Children with TLD were matched to children with DLD on their chronological age and their socio-economic status. Given the relationship between fluid intelligence and analogical reasoning (Chuderski 2015) and the possible weakness of DLD children in fluid IQ (Earle et al. 2017, Vugs et al. 2013), children were also matched on a measure of non-verbal IQ. A control child was selected if he/she had a non-verbal IQ which differed from the DLD child in 8 points maximum. The measure selected combined two important aspects of fluid intelligence, i. e., matrices solving and working memory (Chuderski 2015). The two groups did not differ on their non-verbal IQ score nor on their memory span. As the non-verbal intelligence of our participants was controlled, we will be able to disentangle an analogical reasoning impairment from a weakness in general fluid intelligence. Choosing a measure containing a matrices task could be surprising, as matrices tasks, notably the Raven's Progressive Matrices (Raven 2003), have been related to analogical reasoning. However, several studies have shown that participants from different population matched on a matrices task obtained different results in other analogical tasks (Denaes and Berger 2014, Krawczyk et al. 2014, Sahyoun et al. 2009). Matrices do not seem to assess as precisely as other analogical tasks analogical reasoning ability. Thus, matching our participants on a measure including a matrices task should not prevent the identification of an analogical reasoning impairment in 
DLD. Finally, both groups were significantly different on all standardized measures of language (see table 1).

We received the informed consent of parents for all the children. The local research ethics committee approved the study, which was carried out in accordance with the guidelines of the Helsinki Declaration.

-Insert table 1 about here-

\section{Stimulus material}

Analogical mapping was investigated by the means of two pattern-based abstraction tasks: a sequential task and an equivalent simultaneous task. Each task was composed of 54 items. An item was composed of three three-unit sequences: two reference sequences (considered as priming sequences) and one test sequence (considered as a target sequence). The sequences were composed of three pictures which had little semantic content. These pictures came from the study of Kroll and Potter (1984). In order to select the pictures that had the least semantic content, a questionnaire with 35 pictures were distributed to 70 adults. By means of a Likert scale from 1 (Strongly disagree) to 5 (Strongly agree), the adults have to determine if pictures looked like a real object. After analyses, we have kept 11 pictures for which all the adults had principally responded "Strongly disagree" or "Disagree". We have deliberately chosen pattern-based abstraction which does not require linguistic knowledge or knowledge of form-meaning pairings in order to test the basic processes involved in analogical mapping (i.e. to infer a relational structure without taking account of the meaning and to generalize this structure to new items). In these tasks, children had to choose two pictures among four available pictures (two different shapes, each of them of two different colors) for completing the third sequence (the test sequence). Children had to complete the test sequence according to the particular logical relation existing between the units of the two sequences being analogized (the two reference sequences). For studying the influence of processing load on analogical mapping, we considered two characteristics: the perceptual similarity (with vs without) and the presentation format of the units within the sequences (sequential vs simultaneous). These two factors influence processing load notably by the additional demands imposed on working memory, i. e. the ability to temporarily store and manipulate information for complex cognitive tasks (Baddeley 1992). The identification of perceptual similarity does not require any working memory resources, but the identification of relational similarity without perceptual similarity necessitates an efficient working memory ability (Waltz et al. 2000). Moreover, sequential presentation constrains participants to maintain units in memory and to mentally manipulate them in order to solve the analogy. Items without perceptual support, as well as items presented sequentially, may therefore increase the working memory demands and so the processing load of the task. 
To analyze the role of perceptual similarity on children's performance, we created items with and without perceptual support in each task (see figure 1). As we have already seen it, perceptual similarity can help children identify relational similarity and so improve their analogical reasoning ability (Gentner and Markman 1997). In contrast, analogical items with no perceptual similarity can be more difficult to solve, especially for DLD children, increasing working memory demands and processing load (Leroy et al. 2012, Waltz et al. 2000). The 27 items with perceptual support contained three sequences (two reference sequences and one test sequence) which shared pictures with a similar shape or a similar color. In contrast, in the items without perceptual similarity, there was no similarity of shape or color between the three sequences. These sequences did not have any shape or color in common, so children had to rely only on relational similarity. Pictures have been colored with one of six colors (red, blue, green, yellow, black and purple). In the same sequence, pictures were discriminable using solely shape and color. Size was not used because this dimension is less salient and thus more cognitively demanding (Marshall 2003).

- Insert figure 1 about here -

In the sequential task, pictures appeared on the screen one after another every 500 milliseconds. Visual cues stayed on the screen at the same place as the picture to indicate the length of the reference sequences. Both reference sequences were presented twice before the test sequence appeared. Children were asked to complete the test sequence by choosing the two pictures that 'went best' among four possible responses (see figure 2).

In contrast, in the simultaneous task, the first reference sequence appeared first on the screen. The second reference sequence appeared under the first sequence on the screen two seconds later. The first picture of the test sequence also appeared under the two reference sequences two seconds after the second reference sequence. The three sequences stayed on the screen and the children had no time limit to complete the test-sequence (see figure 3 ).

- Insert figures 2 and 3 about here-

\section{Procedure}

Participants were tested individually in a separate room. A session of 40 minutes was proposed for each of the two tasks. E-Prime (Schneider et al. 2002) was the computer software used to run the experiment.

Before the experimental tasks, children were administrated a visual discrimination task 
in order to ensure that the potential difficulties of children with DLD were not caused by poor discrimination of the stimuli. Children had to judge verbally if two pictures, which were the same than those used in the experimental tasks, were identical or not. The threshold of correct responses was fixed at $80 \%$. If a child did not reach this threshold, the experimental tasks were not proposed. However, no child had to be excluded after the discrimination task.

For completing the test sequence, children had to choose two pictures among four available ones. Each possible response was linked to a specific keyboard key. A typing mask, on which pictures corresponding to the four possible solutions were placed next to the associated key, was placed on the keyboard. Prior to the testing phase, children were submitted to training trials, which allowed them to become familiar with the task and the instructions. Following the practice trials, children were presented with the testing task. In order to maintain the children's attention during the whole testing phase, the task was inserted inside a story and rewards were awarded every six items.

In the two tasks, the presentation of the items was randomized. Moreover, the order of the two tasks was counterbalanced across participants. Half of the participants began with the sequential task whereas the other half began with the simultaneous task.

\section{Results}

The dependent variable was the total number of items successfully completed. Performances showed neither floor nor ceiling effects, with skewness estimates being comparable and remaining in the range of 2 standard errors. No child was excluded from the analyses. The results are shown in table 2.

- Insert table 2 about here -

A repeated-measure analysis of variance, 2 (Presentation format: sequential vs. simultaneous) X 2 (Perceptual support: with vs. without) with the group (DLD vs. TLD) as independent variable, revealed a main effect of Group $\left(F(1,38)=22.14, p<.001, \eta^{2}{ }^{2}=.37\right)$, with poorer performances for children with DLD than for their age-matched peers. A significant main effect of Presentation format was revealed $\left(F(1,38)=18.06, p^{<.001,} \eta^{2}=.32\right)$. All the children performed less well for the sequential task than for the simultaneous task. Interestingly, no significant interaction between Presentation format and Group was found $(F(1,38)=0.03$, $p=.87, \eta^{2}=.001$ ). Children with DLD had poorer performance than children with TLD but their performance did not seem to be more affected by sequential presentation than by simultaneous presentation (see figure 4). 
A significant main effect of Perceptual support was revealed $(F(1,38)=9.49, p=.004$, $\eta^{2}{ }^{2}=.20$ ). Children performed better items with perceptual similarity than items without perceptual similarity. A significant interaction between Perceptual support and Group was revealed $\left(F(1,38)=9.49, p=.004, \eta_{p}^{2}=.20\right)$. While a significant effect appeared between items with and without perceptual similarity in children with $\operatorname{DLD}\left(F(1,19)=31.79, p<.001, \eta^{2}=.63\right)$, such an effect was not observed in children with TLD $\left(F(1,19)<0.001, p=1.00, \eta_{p}{ }^{2}=.00\right)$ (see figure 5). Finally, the interaction between Perceptual support, Presentation format and Group was not significant $\left(F(1,38)=0.25, p=.62, \eta^{2} p=.006\right)$.

\section{- Insert figure 5 about here -}

Then, we conducted a repeated-measure analysis of covariance, 2 (Presentation format: sequential vs. simultaneous) X 2 (Perceptual support: with vs. without) with the group (DLD vs. TLD) as independent variable and the morphosyntax measures in production and comprehension as covariates. We decided to add these measures given the link existing between analogical reasoning and language development, and more precisely between pattern-based abstraction and morphosyntax acquisition. We wanted to see if the group difference could be explained by the language abilities of participants. Results showed that the effect of Group disappeared, $F(1,38)=1.49, p=.30, \eta^{2}{ }^{2}=.038$. The main effect of Presentation format was still significant, $F(1,38)=18.06, p<.001, \eta^{2}=.32$, as well as the effect of Perceptual support, $F(1,38)=9.49, p=.004, \eta^{2}=.20$. Interestingly, the interaction between Perceptual support and Group was also still significant, $F(1,38)=9.49, p=.004, \eta^{2}=.20$ : the effect of Perceptual support was significant for the children with DLD, $F(1,19)=31.79, p<.001, \eta^{2}{ }^{2}=.63$, but not for the children with TDL, $F(1,19)<.001, p=1.00, \eta^{2}=.00$. No other interaction was significant (all $p>.10)$.

\section{Discussion}

The goal of this study was to investigate analogical mapping in children with DLD, and the influence of processing load on their performance, using two different ways of loading (presentation format or perceptual support). Overall, the results show that children with DLD performed less well than their age-matched peers with TLD: in a non-linguistic situation, pattern-based abstraction, which depends on analogical mapping, is less efficient in children with DLD. These results corroborate results obtained in previous studies using linguistic or non-linguistic items without semantic content (Leroy et al. 2012, Leroy et al. 2014a). Krzemien et al. (2017) also supported this conclusion of an analogical reasoning impairment in children 
with DLD compared to age-matched peers, with a task using real-life scenes and varying along relational complexity and perceptual distraction levels. Moreover, children with DLD were more influenced by the increase of relational complexity compared to their language-matched peers, which mean that their performance diminishes with the increase in cognitive load. However, our group effect disappeared when we entered our morphosyntax measures as covariates, which means that the analogical reasoning impairment of DLD children could be due to their morphosyntax difficulties.

Moreover, our results were different according to the way that we manipulated cognitive load. When testing presentation format (sequential task vs simultaneous task), the performance of children with DLD was poorer than the performance of children with TLD, but the difference between the two groups was not more marked in the sequential task than in the simultaneous task. Gabriel et al. (2015) obtained the same effect of presentation format in their task: DLD and TLD children presented the same difference in performance between the simultaneous and sequential conditions in a visuo-spatial pattern task. The performance of children with DLD was not more affected by the sequential presentation of patterns. When testing perceptual support, our results show the sensitivity of children with DLD to the presence of perceptual similarity, even when their morphosyntax abilities were controlled. The difference between the two groups was more marked in the items without perceptual similarity compared to the items with perceptual similarity. In fact, our results show that children with TLD were not sensitive to the existence of perceptual similarity, as their results were similar with and without it. The difference between our results for the two variables suggests that, although analogical mapping is strongly correlated with processing load (Gentner and Smith 2012) and our two variables involved processing load, the underlying tasks were different and had different effect on children with DLD.

In the present study, the children were matched on age but also on their reverse span, which means that they had similar working memory capacity. Despite the complexity of separating working memory from analogical reasoning (Thibaut et al. 2010), we can think that the well-known working memory difficulties of children with DLD (Marton and Schwartz 2003) were not the explanation for their poorer performance. We can also argue that the difficulties of children with DLD in these tasks did not result from a problem with sequential processing per se. Clearly, diminishing the memory constraints by means of simultaneous presentation was helpful for all children. On the one hand, they did not have to overload their working memory, and on the other hand, they could look back at the reference elements, using them as if they had multiple samples to work with. The difficulties of DLD children with analogy are therefore not related with sequential presentation and the overload in working memory which is caused by it.

The differences found in children with DLD for the perceptual support variable must have a different cause. One explanation could be a difference in the process used by the children. Indeed, reasoning by analogy is the result of a developmental trajectory characterized by two different strategies (Thibaut et al. 2010): while younger children use the first strategy, giving priority to perceptual commonalities, older children use the second strategy and give 
priority to relational similarity. In our tasks, the items with perceptual similarity can be solved by using preferentially the first strategy. However, using this first strategy is not sufficient to solve the items without perceptual similarity and in this case, children with DLD seem to have difficulty using the second strategy. Processing relational matches requires more processing capacity than processing simple object matches (Waltz et al. 2000), and children with DLD have processing limitations (Im-Bolter et al. 2006). Perceptual matches supporting relational similarities may help them by decreasing the processing load of the analogical task. Moreover, inhibitory control, which permits children to suppress object matches in favor of relational matches (Richland and Morrison 2010), could influence the results. Children with DLD have inhibitory disorders (Marton et al. 2007), which could explain their difficulties in ignoring object matches so as to focus on relational matches. Krzemien et al. (2017) have confirmed the influence of working memory and inhibition on analogical reasoning, including in DLD children. It is therefore possible that a poor inhibition level in children with DLD prevents them from focusing on relational similarities instead of perceptual ones, making their performance even poorer in items without perceptual commonalties.

Even if the group effect disappeared when morphosyntax abilities were controlled, children with DLD have analogical reasoning difficulties compared to age-matched peers, and their dependence to perceptual support is even present beyond their language difficulties. Moreover, analogical reasoning and language share a mutual influence, one allowing for the development of the other (Gentner and Chritie 2010). It is therefore possible that language disorders cause an analogical reasoning impairment, which in turn hinders language development in children with DLD. Analogical reasoning, and so analogical mapping, is indeed required for lexical categorization and word extension (Gentner et al. 2011, Gentner and Namy 1999), as well as for the generalization of morphemes and syntactic structures (Bybee 2010). Many studies have shown that these aspects, and especially the productive use of construction schemas, are altered in children with DLD (Collisson et al. 2015, Riches et al. 2006). It is therefore more than plausible that the analogical mapping deficit observed in children with DLD causes their difficulties in the generalization of construction schemas, and so their language disorders. Furthermore, it does not seem to be the transient nature of speech which causes their difficulty with analogy, as they are as impaired by sequential presentation as their peers. The increase of processing load and the additional demands on working memory caused by sequential presentation do not account for the difficulty of DLD children in analogical mapping. This has important implication for language learning, as the core difficulty of DLD children does not seem to be the transitory aspect of language and the overload of working memory which is caused by it, but the structural alignment process per se. However, children with DLD are more sensitive to the absence of perceptual similarity. This difficulty in processing relational similarity alone may also have consequences for language acquisition. Children with DLD could indeed have more trouble abstracting and generalizing construction schemas when the forms which have to be mapped do not share any perceptual similarity, i. e. do not have any phonemes or words in common. Thus, Leroy et al. (2014b) found that children with DLD performed worse than their language-matched peers in a priming task, especially when the prime sentence and the target sentence did not have any words in common. It is therefore possible that the difficulty of children with DLD in identifying relational similarity 
without perceptual similarity hinders them from generalizing linguistic structures on the basis of dissimilar forms.

\section{Conclusions}

In this study, we evaluated analogical mapping in children with DLD thanks to a patternbased abstraction task. The analyses showed that children with DLD have poorer performance than their age-matched peers without language disorders, but similar global performance when morphosyntax abilities were controlled. We also manipulated the cognitive load of the task: sequential presentation of the items lead to worse performance than simultaneous presentation in all participants. However, the absence of perceptual similarities within an item impaired the performance of children with DLD only, even when morphosyntax abilities were controlled. These difficulties could be due to the deficit of processing capacity or of inhibitory control observed in children with DLD.

The results obtained are compatible with the idea that the language difficulties of children with DLD cause an analogical reasoning impairment, which in turn hinders language development and more precisely the mechanism of generalization. However, other studies are needed to confirm this hypothesis, as there is no sufficiently strong evidence yet to claim that a clear link between language disorders and analogical reasoning exists. Nonetheless, our tasks were pattern-based abstraction tasks in which children had to discover a relational structure, and pattern-based abstraction plays a role in category-based generalization, which is fundamental to language productivity (Gómez and Gerken 2000). Thus, our results indicate that pattern-based abstraction, a basic key process of analogical mapping and of language development, is impaired in children with DLD. However, the influence of analogical reasoning on language development may be more complex, consisting in a mutual influence or implying other external factors. More investigations in this theoretical framework would be interesting to clarify the role of analogical reasoning on language development, and to understand better language disorders in children with DLD.

\section{Acknowledgment}

This work was supported by the Fonds de la Recherche Scientifique - FNRS (Belgium). We thank all the children who participated in this study, as well as the language therapists and the teachers for their support. We are grateful to Aurélie Pabiot for her assistance in the data collection.

\section{References}

BADDELEY, A., 1992, Working memory. Science, 255(5044), 556-559. 
BYBEE, J., 2010, Language, Usage and Cognition (Cambridge, UK: Cambridge University Press).

CHEN, Z., 1996, Children's analogical problem solving: The effects of superficial, structural, and procedural similarity. Journal of Experimental Child Psychology, 62, 410-431.

CHILDERS, J. B., 2011, Attention to multiple events helps two-and-a-half-year-olds extend new verbs. First Language, 31(1), 3-22.

CHRISTENSEN, R. V., and HANSSON, K., 2012, The use and productivity of past tense morphology in specific language impairment: An examination of Danish. Journal of Speech, Language, and Hearing Research, 55(6), 1671-1689.

CHRISTIE, S., and GENTNER, D., 2010, Where hypotheses come from: Learning new relations by structural alignment. Journal of Cognition and Development, 11(3), 356373.

CHUDERSKI, A., 2015, Why people fail on the fluid intelligence tests. Journal of Individual Differences, 36(3), 138-149.

COLLISSON, B. A., GRELA, B., SPAULDING, T., RUECKL, J. G., and MAGNUSON, J. S., 2015, Individual differences in the shape bias in preschool children with specific language impairment and typical language development: theoretical and clinical implications. Developmental Science, 3, 373-388.

CONTI-RAMSDEN, G., and WINDFUHR, K., 2002, Productivity with word order and morphology: A comparative look at children with SLI and children with normal language abilities. International Journal of Language \& Communication Disorders, 37(1), 17-30.

DENAES, C., AND BERGER, J. L., 2014, Analogical reasoning in adolescents with intellectual disability: effects of external memories and time processing. Journal of Research in Special Educational Needs, 14(2), 82-95.

EARLE, F. S., GALLINAT, E. L., GRELA, B. G., LEHTO, A., and SPAULDING, T. J., 2017, Empirical implications of matching children with specific language impairment to children with typical development on nonverbal IQ. Journal of learning disabilities, 50(3), 252-260.

GABRIEL, A., MEUlEMANS, T., PARISSE, C., and MAILlART, C., 2015, Procedural learning across modalities in French-speaking children with developmental language disorders. Applied Psycholinguistics, 36(3), 747-769.

GENTNER, D., 1983, Structure-mapping: A theoretical framework for analogy. Cognitive science, 7(2), 155-170.

GENTNER, D., 1988, Metaphor as structure mapping: The relational shift. Child Development, 
59(1), 47-59.

GENTNER, D., ANGGORO, F. K., and KLIBANOFF, R. S., 2011, Structure mapping and relational language support children's learning of relational categories. Child Development, 82(4), 1173-1188.

GENTNER, D., and COLHOUN, J., 2010, Analogical processes in human thinking and learning. In B. Glatzeder, V. Goel, and A. Müller (eds), Towards a Theory of Thinking (Berlin, Germany: Springer Berlin Heidelberg), pp 35-48.

GENTNER, D., and MARKMAN, A. B., 1997, Structure mapping in analogy and similarity. American Psychologist, 52(1), 45-56.

GENTNER, D., and NAMY, L. L., 1999, Comparison in the development of categories. Cognitive Development, 14(4), 487-513.

GENTNER, D., and NAMY, L. L., 2006, Analogical processes in language learning. Current Directions in Psychological Science, 15(6), 297-301.

GENTNER, D., and SMITH, L., 2012, Analogical reasoning. In V. Ramachandran (ed), Encyclopedia of Human Behavior (2nd ed.) (Oxford, UK: Elsevier), pp. 130-136.

GENTNER, D. and SMITH, L. A., 2013, Analogical learning and reasoning. In D. Reisberg (ed), The Oxford Handbook of Cognitive Psychology (Oxford, UK: Oxford University Press), pp. 668-681.

GOLDBERG, A. E., 2003, Constructions: A new theoretical approach to language. Trends in cognitive sciences, 7(5), 219-224.

GÓMEZ, R. L., and GERKEN, L., 2000, Infant artificial language learning and language acquisition. Trends in Cognitive Sciences, 4(5), 178-186.

HSU, H. J., and BISHOP, D. V., 2010, Grammatical difficulties in children with specific language impairment: Is learning deficient? Human development, 53(5), 264-277.

IM-BOLTER, N., JOHNSON, J., and PASCUAL-LEONE, J., 2006, Processing limitations in children with developmental language disorders: The role of executive function. Child Development, 77(6), 1822-1841.

KHOMSI, A., 2001, ELO: évaluation du langage oral (Paris, France: ECPS).

KRAWCZYK, D. C., KANDALAFT, M. R., DIDEHBANI, N., ALLEN, T. T., MCCLELLAND, M. M., TAMMINGA, C. A., and CHAPMAN, S. B., 2014, An investigation of reasoning by analogy in schizophrenia and autism spectrum disorder. Frontiers in Human Neuroscience, 8, 517.

KROLL, J. F., and POTTER, M. C., 1984, Recognizing words, pictures, and concepts: A 
comparison of lexical, object, and reality decisions. Journal of Verbal Learning and Verbal Behavior, 23, 39-66.

KRZEMIEN, M., JEMEL, B., and MAILLART, C., 2017, Analogical reasoning in children with developmental language disorders: Evidence from a scene analogy task. Clinical Linguistics \& Phonetics, 31, 573-588.

LEONARD, L. B., DAVIS, J., and DEEVY, P., 2007, Phonotactic probability and past tense use by children with developmental language disorders and their typically developing peers. Clinical Linguistics \& Phonetics, 21, 747-758.

LEROY, S., MAILLART, C., and PARISSE, C., 2014a, Analogical mapping across modalities in children with developmental language disorders (DLD). Research in Developmental Disabilities, 35(9), 2158-2171.

LEROY, S., PARISSE, C., and MAILLART, C., 2012, Analogical reasoning in children with developmental language disorders. Clinical Linguistics \& Phonetics, 26(4), 380-395.

LEROY, S., PARISSE, C., and MAILLART, C., 2014b, Le manque de généralisation chez les enfants dysphasiques: Une étude longitudinale. ANAE, 131, 1-9.

MARTON, K., KELMENSON, L., and PINKHASOVA, M., 2007, Inhibition control and working memory capacity in children with DLD. Psychologia: An International Journal of Psychology in the Orient, 50(2), 110-121.

MARTON, K., and SCHWARTZ, R. G., 2003, Working memory capacity and language processes in children with developmental language disorders. Journal of Speech, Language, and Hearing Research, 46(5), 1138-1153.

MASTERSON, J. J., EVANS, L. H., and ALOIA, M., 1993, Verbal analogical reasoning in children with language-learning disabilities. Journal of Speech and Hearing Research, $36,76-82$.

MUSSWEILER, T., and EPSTUDE, K., 2009, Relatively fast! Efficiency advantages of comparative thinking. Journal of Experimental Psychology: General, 138(1), 1-21.

RAVEN, J., 2003, Raven progressive matrices. In R. S. McCallum (ed), Handbook of Nonverbal Assessment (Boston, MA: Springer), pp. 223-237.

RICHES, N., FARAGHER, B., and CONTI-RAMSDEN, G., 2006, Verb schema use and input dependence in 5-year-old children with developmental language disorders (DLD). International Journal of Language \& Communication Disorders, 41(2), 117-135.

RICHLAND, L. E., and MORRISON, R. G., 2010, Is analogical reasoning just another measure of executive functioning? [Comment/Reply]. Frontiers in Human Neuroscience, 4, 180.

RICHLAND, L. E., MORRISON, R. G., and HOLYOAK, K. J., 2006, Children's development 
of analogical reasoning: Insights from scene analogy problems. Journal of Experimental Child Psychology, 94(3), 249-273.

RICHLAND, L. E., and SIMMS, N., 2015, Analogy, higher order thinking, and education. WIREs Cognitive Science, 6, 177-192.

ROEHM, M. L., and STERNTHAL, B., 2001, The moderating effect of knowledge and resources on the persuasive impact of analogies. Journal of Consumer Research, 28(2), 257-272.

SAHYOUN, C. P., SOULIERES, I., BELliVEAU, J. W., MOTTRON, L., and MODY, M., 2009, Cognitive differences in pictorial reasoning between high-functioning autism and Asperger's syndrome. Journal of Autism and Developmental Disorders, 39(7), 10141023.

SCHNEIDER, W., ESCHMAN, A., and ZUCCOLOTTO, A., 2002, E-Prime (Version 2.0) (Pittsburgh, PA: Psychology Software Tools Inc).

SCHWARTZ, R. G., 2009, Developmental language disorders. In R. G. Schwartz (ed), Handbook of Child Language Disorders (New York, NY: Psychology Press), pp. 3-43.

SKIPP, A., WINDFUHR, K. L., and CONTI-RAMSDEN, G., 2002, Children's grammatical categories of verb and noun: A comparative look at children with developmental language disorders (DLD) and normal language (NL). International Journal of Language \& Communication Disorders, 37(3), 253-271.

THIBAUT, J.-P., FRENCH, R., and VEZNEVA, M., 2010, The development of analogy making in children: Cognitive load and executive functions. Journal of Experimental Child Psychology, 106(1), 1-19.

TOMAS, E., DEMUTH, K., and PETOCZ, P., 2017, The role of frequency in learning morphophonological alternations: Implications for children with Specific Language Impairment. Journal of Speech, Language, and Hearing Research, 60(5), 1316-1329.

TOMASELLO, M., 2009, The usage-based theory of language acquisition. In E. L. Bavin (ed), The Cambridge Handbook of Child Language (Cambridge, UK: Cambridge University Press), pp. 69-88.

VUGS, B., CUPERUS, J., HENDRIKS, M., and VERHOEVEN, L., 2013, Visuospatial working memory in specific language impairment: A meta-analysis. Research in Developmental Disabilities, 34(9), 2586-2597.

WALTZ, J. A., LAU, A., GREWAL, S. K., and HOLYOAK, K. J., 2000, The role of working memory in analogical mapping. Memory \& Cognition, 28(7), 1205-1212.

WECHSLER, D., and NAGLIERI, J., 2009, Echelle non verbale d'intelligence de Wechsler (Montreuil, France: ECPA). 
Table 1. Age, IQ and standardized scores for language assessment measures for the DLD and the TLD groups.

\begin{tabular}{|l|c|c|c|c|c|c|c|}
\cline { 2 - 8 } \multicolumn{1}{c|}{} & \multicolumn{3}{c|}{ DLD (n=20) } & \multicolumn{3}{c|}{ TLD (n=20) } & \multicolumn{1}{c|}{} \\
\hline Variable & M & SD & Range & M & SD & Range & t \\
\hline Age (in months) & 122 & 18.91 & $85-157$ & 121.35 & 17.11 & $89-153$ & 0.11 \\
\hline PIQ (WNV) & 98.55 & 9.21 & $87-120$ & 98.75 & 9.01 & $86-113$ & -0.07 \\
\hline Visual memory Span & & & & & & & \\
\hline Reverse order (WNV) - Raw score & 5.95 & 1.53 & $3-9$ & 6.2 & 1.36 & $3-8$ & -0.54 \\
\hline LLO & & & & & & & \\
\hline Lexical production (Raw score) & 29.8 & 5.89 & $18-38$ & 39.45 & 5.91 & $27-46$ & $-5.17^{* * *}$ \\
\hline Word repetition (Raw score) & 21.65 & 7.12 & $9-31$ & 31.85 & 0.36 & $31-32$ & $-6.4^{* * * *}$ \\
\hline Utterances production (Raw score) & 13.1 & 4.79 & $5-24$ & 22.8 & 2.21 & $17-25$ & $-8.22^{* * *}$ \\
\hline Sentence comprehension (Raw & 19.05 & 5.03 & $9-28$ & 25.2 & 4.28 & $17-30$ & $-4.16^{* * *}$ \\
\hline score) & & & & & & & \\
\hline
\end{tabular}

Note. PIQ= Performance Intelligence Quotient ; M= Mean ; SD= Standard Deviation ; *** $\mathrm{p}<0.001$ 
Table 2. Mean number (and standard deviation) of correct responses as a function of perceptual support and modality across the two groups.

\begin{tabular}{|l|c|c|c|c|}
\cline { 2 - 5 } \multicolumn{1}{c|}{} & \multicolumn{2}{c|}{ Sequential presentation } & \multicolumn{2}{c|}{ Simultaneous presentation } \\
\cline { 2 - 5 } \multicolumn{1}{c|}{} & $\begin{array}{c}\text { With } \\
\text { perceptual } \\
\text { support }\end{array}$ & $\begin{array}{c}\text { Without } \\
\text { perceptual } \\
\text { support }\end{array}$ & $\begin{array}{c}\text { With } \\
\text { perceptual } \\
\text { support }\end{array}$ & $\begin{array}{c}\text { Without } \\
\text { perceptual } \\
\text { support }\end{array}$ \\
\hline DLD group & $17.65(5.14)$ & $16.10(5.06)$ & $19.80(4.88)$ & $18.75(5.25)$ \\
\hline TLD group & $22.20(3.81)$ & $22.20(4.37)$ & $24.80(1.58)$ & $24.80(1.77)$ \\
\hline
\end{tabular}


Figure 1. Examples of the items

Examples of sequences with perceptual support(with common color(s) between sequences)

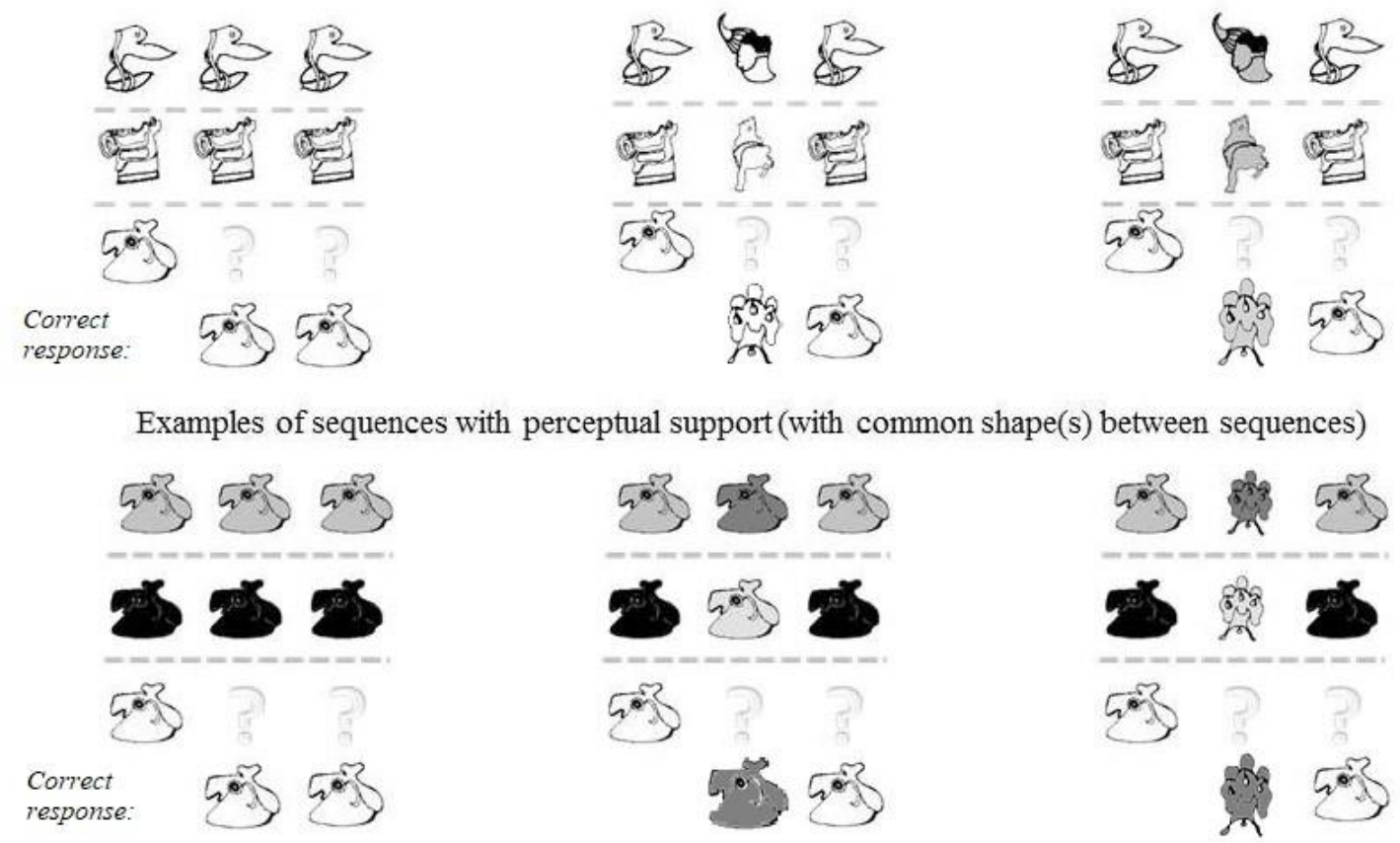

Examples of sequences without perceptual support (neither common color nor common shape between sequences)

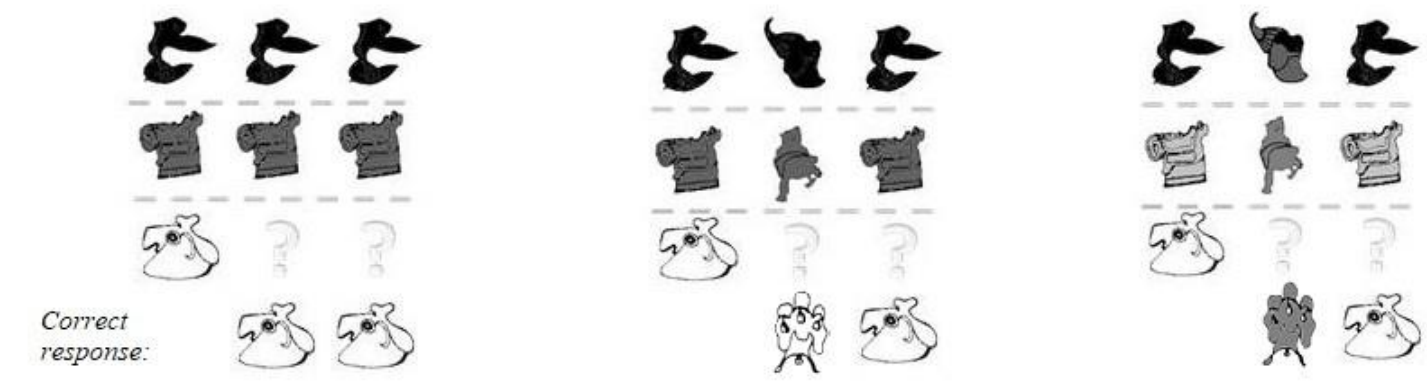


Figure 2. Presentation of the items in the sequential task

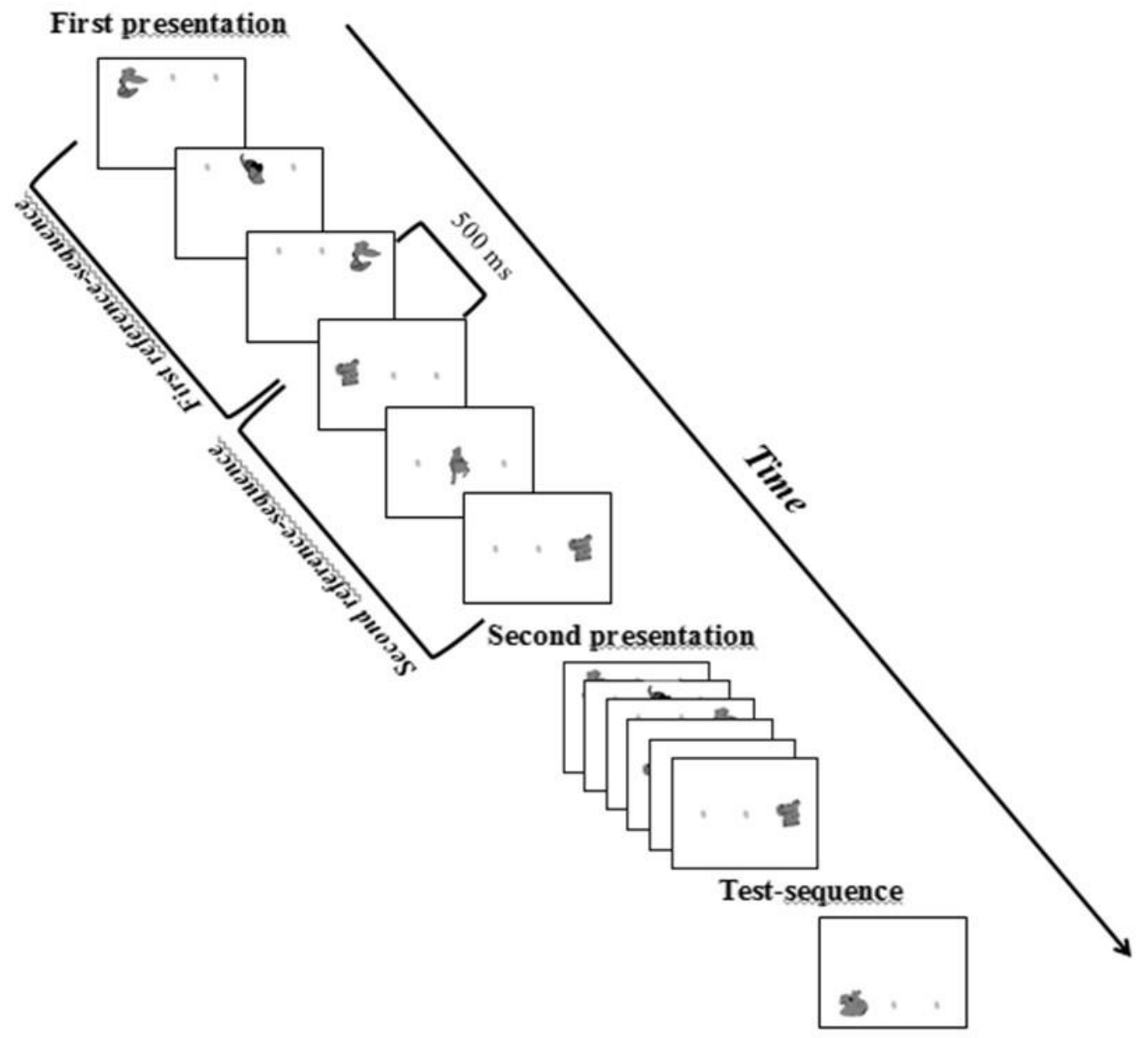


Figure 3. Presentation of the items in the simultaneous task

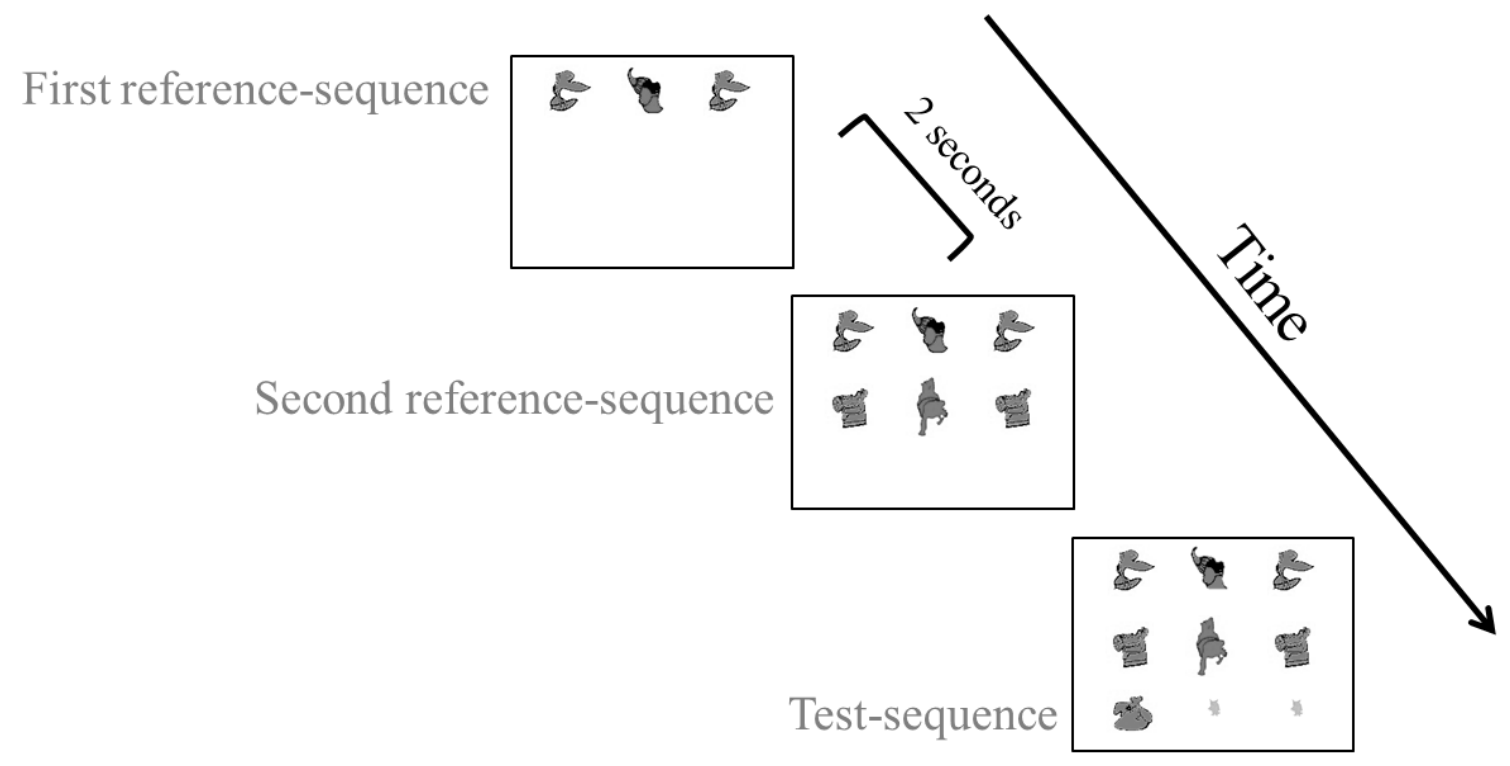


Figure 4. Number of correct responses as a function of the modality of presentation across the two groups. Note: Bars represent the $95 \%$ confidence intervals

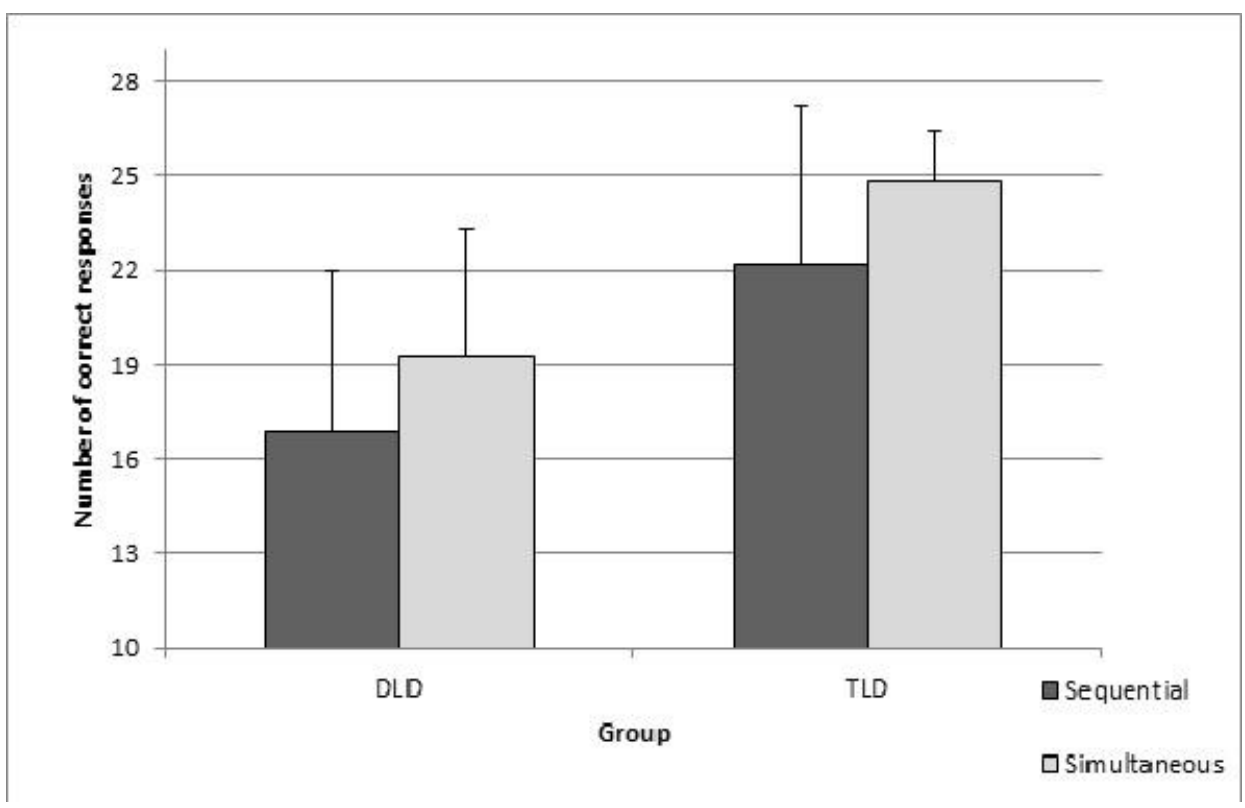


Figure 5. Number of correct responses as a function of the presence or not of perceptual similarity across the two groups. Note: Bars represent the $95 \%$ confidence intervals

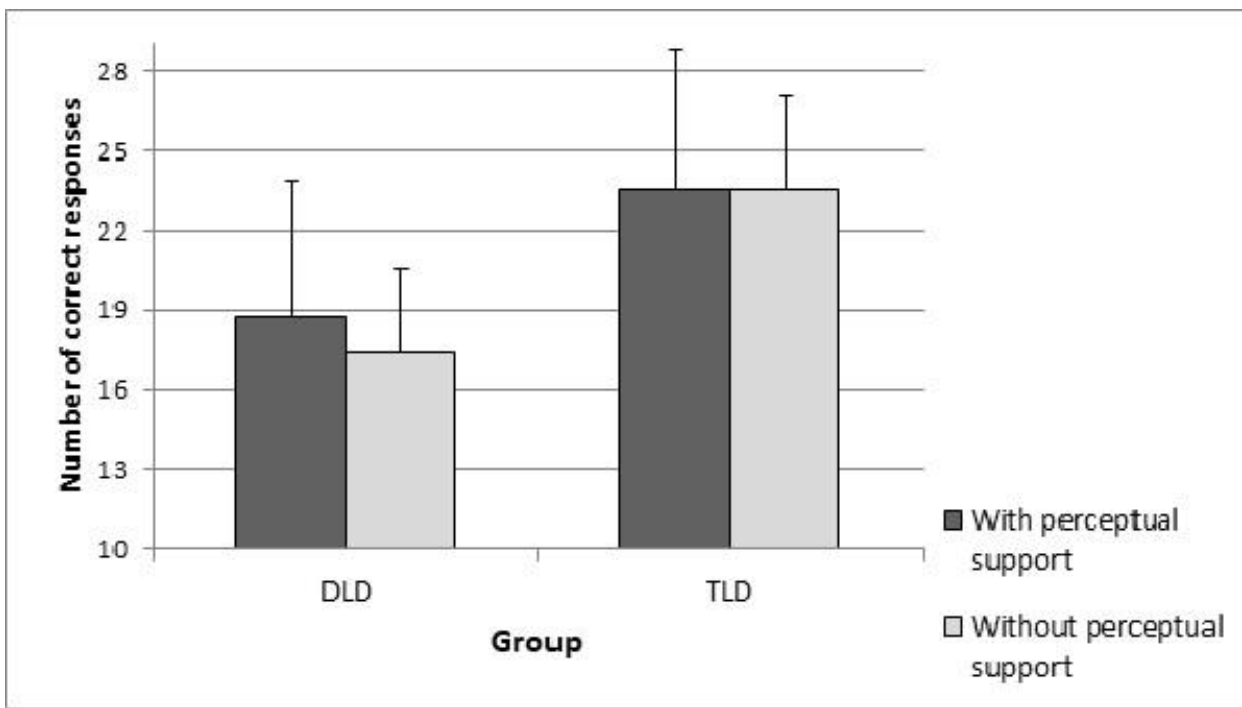

